\title{
Adaptive Tuning of Large-Signal Resonant Circuits Using Phase-Switched Fractional Capacitance
}

\author{
W. Redman-White, Fellow, IEEE, H. Kennedy, R. Bodnar, Member, IEEE, and T. Lee
}

\begin{abstract}
Inductively-coupled systems used in applications such as RFID and wireless power often require high $Q$ factor resonant transmitters to maximise the magnetic field and achieve high overall efficiency. However, these are sensitive to environmental detuning as well as component tolerances. Existing methods for accurate tuning require search algorithms, usually requiring the suspension of normal operation in order to calibrate the resonant inductor-capacitor circuit, thus reducing power throughput and increasing system complexity.

We describe here how zero-voltage switched fractional capacitance techniques may be used to achieve continuous and real-time adaptive tuning of large-signal resonant inductorcapacitor circuits. Minimal additional circuitry is required and tuning is maintained without disrupting normal operation. Many variants are possible for the implementation of the system, and some trade-offs relating to the available tuning range and operating voltages are analysed for two alternative topologies. Experimental results are presented for a $125 \mathrm{kHz}$ demonstration system.
\end{abstract}

Index Terms - adaptive tuning, inductive coupling, resonant circuits, RFID, wireless power

\section{INTRODUCTION}

W IRELESS power transfer has a range of applications, from low-power RFID [1] and similar security systems, to high-power vehicle charging [2]. In most systems, efficient operation necessitates the induction of a significant EMF in the receiver circuit, achieved by generating a large magnetic field at the transmitter. This is often done by resonating the transmit coil with a capacitor, and ensuring that there are low losses to permit the largest possible current flow, and hence magnetic field, for a given drive voltage.

In order to achieve the maximum current, the LC circuit must be driven precisely at the resonant frequency. The absolute values of the LC circuit components may, however, vary significantly due to manufacturing tolerances, and also due to changing environmental effects, altering the resonant frequency [3]. It is therefore necessary to be able to tune the LC circuit such that the resonance and drive frequencies closely match. The tuning requirement is made more demanding by the narrow bandwidth of low-loss LC circuits, due to the high Quality (Q) factor, and hence practical implementations can require very precise tuning to ensure that the LC circuit can always be driven near resonance.

Techniques used for tuning small signal circuits, such as varactors, are not suited to the large voltages often

The authors are with Department of Electronics and Computer Science, University of Southampton, SO17 1BJ, UK

(emails: wrw, hk1g10,r.bodnar, t17e13@ecs.soton.ac.uk). experienced in wireless power applications, and thus large signal LC circuits are commonly tuned to resonance by means of a bank of (often binary weighted) capacitors in parallel with a fixed capacitance as per figure 1 . Since the tuning range is discrete, it is unlikely that exact resonance may always be achieved. The requirement for a higher tuning resolution at high-Q clearly necessitates a greater number of capacitors. For example, if the LC tank circuit components have tolerances of $\pm 20 \%$, then the resonant frequency can be in error from $-9 \%$ to $+12 \%$. With $Q=50$, then to ensure that the tank can be tuned to within its $3 \mathrm{~dB}$ bandwidth, at least 4 selectable binary-weighted capacitors are needed. Each additional capacitor results in greater die or board area as well as increased cost, as more high-voltage switches, pins and other circuitry are required.

Detection of the ideal resonance condition is also important, and this is typically achieved by means of an amplitude measurement of the inductor current or capacitor voltage. The tuning setting can be adjusted and the new amplitude compared with the previous value. A search algorithm is then required to determine which tuning setting yields the highest amplitude of oscillation, although this may require normal operation to be suspended whilst re-calibration takes place. Examples of such systems are given in [5], [6].

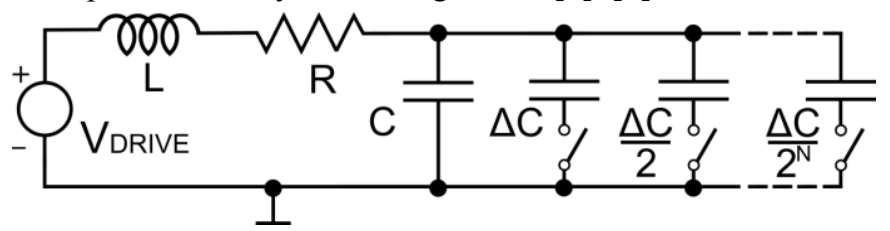

Fig.1: Binary-weighted capacitor method of tuning LC circuits

Alternative methods for tuning LC circuits have been devised [7], [8], but these still rely upon existing methods of resonance detection in order to achieve optimal tuning.

The paper is organised as follows: We first describe how an inductor capacitor circuit may be tuned to resonance using switching of a fractional capacitance element. It is shown that zero-voltage switching may be achieved at resonance with suitable timing. The practical issues of the available tuning range and the switch voltage stress are also discussed. We then show how enforcing this timing regime leads to a method for detecting errors in the resonance condition, and hence can provide the input to an adaptive tuning loop. Experimental results from a hardware demonstration are then given.

\section{Zero Voltage SWITCHING OF FractionaL CAPACITANCE}

Figure 2 shows a topology presented in [9], where a second capacitor $\mathrm{C}_{2}$ is switched in and out of the resonant circuit with a variable duty cycle, creating an average resonant frequency. 
However, no consideration is given to the effect of the switching action on the energy stored in the LC circuit. If the switch is closed when there is significant voltage across it, some power will be dissipated in the loss resistance of the switch, reducing both the $\mathrm{Q}$ factor and the power transferred to the receiver.

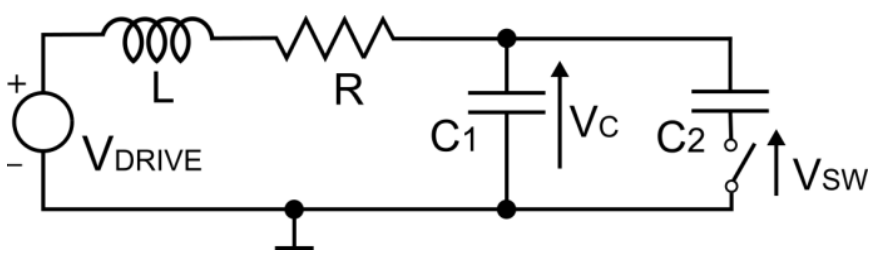

Fig.2: Fractional switched capacitor tuning

The architecture in figure 2 can provide adjustment of the effective resonant frequency while incurring lower switching losses by enforcing zero-voltage switching conditions, i.e. the voltage $v_{s w}(t)$ must be zero whenever the switch is closed, as per the timings in figure 3 . Note that to achieve this, the switching action must occur symmetrically around the peaks and troughs of $\mathrm{v}_{\mathrm{C}}(\mathrm{t})$ when the circuit is at resonance.

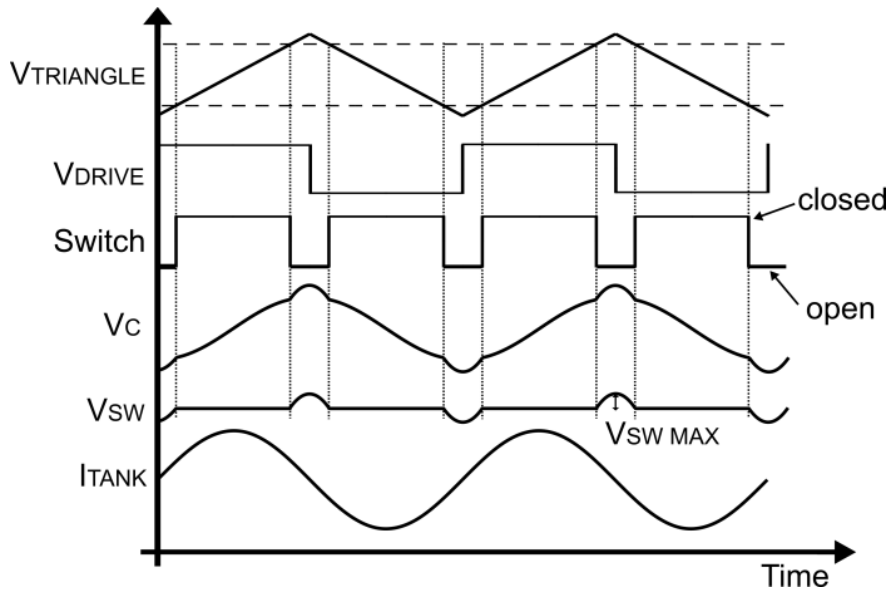

Fig.3a: Ideal timings for zero-voltage switching tuning method at

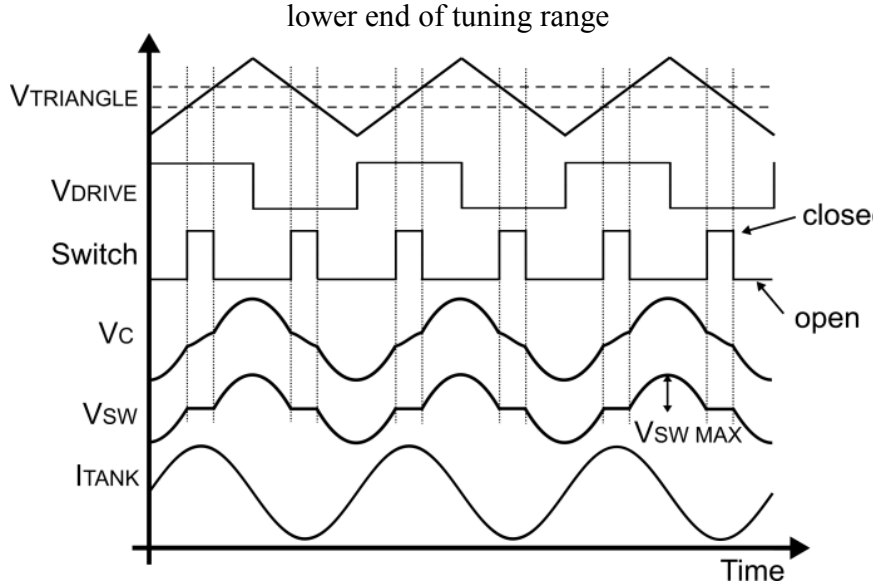

Fig.3b: Ideal timings for zero-voltage switching tuning method at upper end of tuning range

The maximum resonant frequency is clearly given where the capacitor switch is open for the entire cycle, whilst the minimum frequency is where the switch is always closed. Any frequency in between these two values may be achieved by adjusting the phase angle of the switching action about the voltage peak.
Figure 3 shows a triangular-shaped waveform which may be conveniently used to derive switch timings, by comparing the triangle waveform with the $\mathrm{V}_{\text {TUNE }}$ thresholds, in a similar manner to traditional pulse width modulation schemes. Figures $3 \mathrm{a}$ and $3 \mathrm{~b}$ show the typical behaviour of the tuning method towards the extremes of the tuning range, with example waveforms for the cases where $\omega \approx 1 / \sqrt{\mathrm{LC}_{1}}$ and $\omega \approx 1 / \sqrt{\mathrm{L}\left(\mathrm{C}_{1}+\mathrm{C}_{2}\right)}$ respectively.

This tuning method is advantageous over the use of an array of capacitors, since only one additional capacitor and switch are required to achieve resonance at any frequency within the available tuning range, reducing hardware costs. The precision of the tuning with respect to the excitation is determined by the accuracy of both timing generation and specific implementation.

Whilst the capacitor voltage waveform $v_{C}(t)$ is not sinusoidal, a high $\mathrm{Q}$ factor in the LC circuit filters out the harmonics of the capacitor switching action, resulting in a largely sinusoidal current. This means that the radiated harmonics may still be controlled to acceptable levels by using a high Q factor (although this is not significantly different from an existing un-tuned system).

\section{A. $\quad$ Tuning Range}

Provided that the switching action is symmetrical about the ideally-tuned peak capacitor voltage, there is considerable freedom in the choice of the tuning range. For example, if $\pm 20 \%$ combined tolerances are expected to occur in the LC circuit, a tuning range of 1.22:1 may be used to compensate, achieved by making $\mathrm{C}_{1}$ approximately twice the size of $\mathrm{C}_{2}$.

The tuning may be analysed by considering one quadrant of a single period, since it is required for the capacitor switching action to be symmetrical. A lossless LC circuit with initial conditions $\mathrm{v}_{\mathrm{C}}(0)=0$ and $\mathrm{i}(0)=1$ may be used for this purpose. The time domain solution is given by:

$$
\left.\mathrm{i}(\mathrm{t})\right|_{\mathrm{t}<\mathrm{t}_{\mathrm{c}}}=\cos \left(\omega_{\min } \mathrm{t}\right)
$$

At the moment that the switch is opened, a new resonant system is created by removing $\mathrm{C}_{2}$. The initial conditions of the new system are determined by the final conditions of the previous (i.e. the absolute voltage and current values at moment $\left.t=t_{c}\right)$.Substitution of the initial conditions into the general solution for the LC circuit yields:

$$
\left.\mathrm{i}(\mathrm{t})\right|_{\mathrm{t}>\mathrm{t}_{\mathrm{c}}}=\cos \left(\omega_{\min } \mathrm{t}_{\mathrm{c}}\right) \cos \left(\omega_{\max } \mathrm{t}\right)-\frac{\omega_{\min }}{\omega_{\max }} \sin \left(\omega_{\min } \mathrm{t}_{\mathrm{c}}\right) \sin \left(\omega_{\max } \mathrm{t}\right)
$$

The end of the quadrant occurs when $i(t)=0$ and $v_{C}(t)$ has reached a maximum. The time period between the switching action and the end of the quadrant is defined as tp, such that the duration of one quadrant $T_{Q}=t_{C}+t_{p}$. Since the inductor current at the end of the quadrant is zero, $t_{p}$ may be expressed in terms of $t_{C}$ by equating (2) to zero and rearranging:

$$
\mathrm{t}_{\mathrm{p}}=\frac{1}{\omega_{\max }} \tan ^{-1}\left[\frac{\omega_{\max }}{\omega_{\min }} \cot \left(\theta_{\mathrm{c}}\right)\right]
$$

The absolute time value $t_{C}$ may also be normalised as a phase angle of the switching action $\theta_{\mathrm{C}}$, where $0 \leq \theta_{c} \leq \pi / 2$. Since the switching action must be symmetrical, the period of oscillation may be found by multiplying the duration of one 
quadrant by four, and re-written as the modified resonant frequency $\omega_{\mathrm{MOD}}$ :

$$
\omega_{\bmod }=\frac{\pi}{2\left(\left(\theta_{c} / \omega_{\min }\right)+\left(1 / \omega_{\max }\right) \tan ^{-1}\left[\left(\omega_{\max } / \omega_{\min }\right) \cot \left(\theta_{c}\right)\right]\right)}
$$

\section{$B$. \\ Maximum Switch Voltage}

The peak voltage experienced by the capacitor switch when open is given by the product of the drive voltage amplitude and the $\mathrm{Q}$ factor of the LC circuit at the maximum possible effective resonant frequency, i.e. when the switch is open for the entire cycle. To reduce losses, it is preferable to avoid using over-voltage breakdown diodes to protect the switch as part of the normal cycle of operation.

The peak switch voltage reduces as $\theta_{\mathrm{C}}$ is increased, such that the switch opens for a smaller proportion of the cycle. The maximum value may be found from the capacitor voltage at the end of the first quadrant of oscillation. The switch voltage is the integrated current(2) through $\mathrm{C}_{1}$ :

$$
\mathrm{V}_{\mathrm{sw}}=\mathrm{v}_{\mathrm{c}}\left(\mathrm{t}_{\mathrm{p}}\right)=\frac{1}{\mathrm{C}_{1}} \int_{0}^{\mathrm{t}_{\mathrm{p}}} \mathrm{i}(\mathrm{t}) \mathrm{dt}
$$

Since $\mathrm{C}_{2}$ is disconnected, it makes no contribution to the switch voltage. Including the effect of the $\mathrm{Q}$ factor on the amplitude of oscillation results in the inductor current:

$$
\mathrm{I}=\frac{\mathrm{V}_{\text {drive }}}{\mathrm{R}}=\frac{\mathrm{V}_{\text {drive }} \mathrm{Q}_{\max }}{\omega \mathrm{L}}
$$

Equations (2) and (6) may be multiplied to scale the current for $\mathrm{Q}$ factor. The resulting equation may then be integrated as per (3) to yield the peak switch voltage:

$$
\begin{aligned}
\mathrm{V}_{\mathrm{sw}} & =\frac{\mathrm{V}_{\mathrm{drive}} \mathrm{Q}_{\max } \omega_{\max }}{\omega}\left[\cos \left(\theta_{\mathrm{c}}\right) \sin \left(\omega_{\max } \mathrm{t}_{\mathrm{p}}\right)+\right. \\
& \left.+\frac{\omega_{\min }}{\omega_{\max }} \sin \left(\Theta_{\mathrm{c}}\right)\left(\cos \left(\omega_{\max } \mathrm{t}_{\mathrm{p}}\right)-1\right)\right]
\end{aligned}
$$

The breakdown rating associated with $\mathrm{V}_{\mathrm{SW}}$ may therefore be reduced by restricting the minimum value of $\theta_{\mathrm{C}}$, which can be achieved by constraining the range of $\mathrm{V}_{\text {TUNE }}$.

An alternative method of reducing $\mathrm{V}_{\mathrm{SW}}$ is to use the topology in figure 4 , which divides the voltage across the capacitances using $\mathrm{C}_{3}$, such that the observed voltage across the open switch is a fraction of $\mathrm{V}_{\mathrm{TANK}}$. The division ratio between $\mathrm{V}_{\mathrm{SW}}$ and $\mathrm{V}_{\text {TANK }}$ is given by:

$$
\left|\frac{\mathrm{V}_{\mathrm{sw}}}{\mathrm{V}_{\text {tank }}}\right|=\frac{\mathrm{C}_{3}}{\mathrm{C}_{1}+\mathrm{C}_{3}}=\mathrm{n}
$$

Again, $\mathrm{C}_{2}$ makes no contribution to the division ratio for the maximum voltage condition, since it is disconnected. If, for example, we set $C_{1}=C_{2}$, then the tuning range is given by:

$$
\frac{\omega_{\max }}{\omega_{\min }}=\sqrt{2} \frac{\sqrt{\mathrm{C}_{1}+\mathrm{C}_{3}}}{\sqrt{2 \mathrm{C}_{1}+\mathrm{C}_{3}}}
$$

The effectiveness of both switch voltage reduction methods can be compared with respect to the available tuning range in figure 5, for the case where $C_{1}=C_{2}$. In the method of $\theta_{c}$ restriction, a minimum value of $\theta_{\mathrm{C}}$ is being imposed in order to limit $\mathrm{V}_{\mathrm{SW}}$. In the method of capacitive division, the division ratio $n$ is being varied.

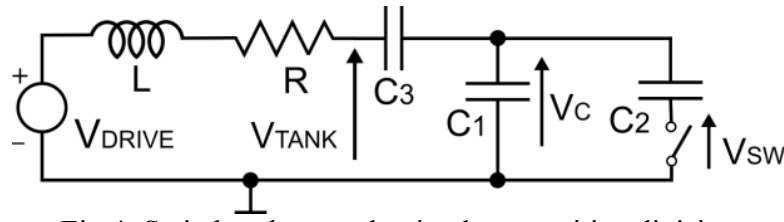

Fig.4: Switch voltage reduction by capacitive division

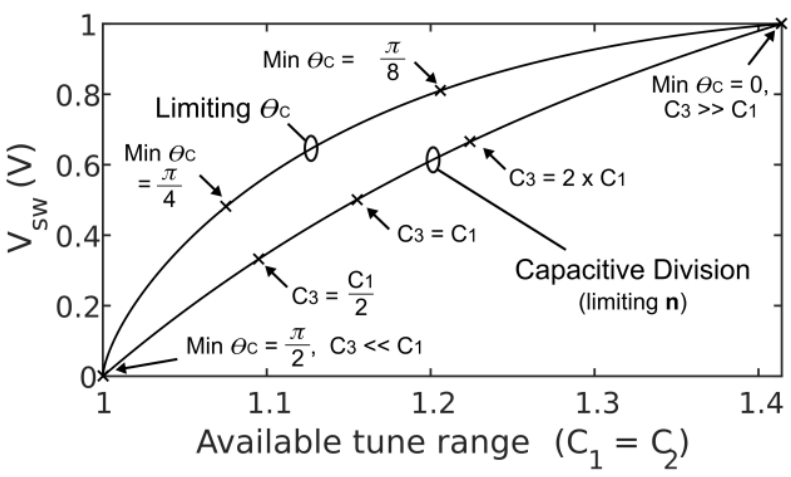

Fig.5: Variation of normalised $V_{\text {sw }}$ compared with normalised tuning range for both methods of switch voltage reduction

It can be seen that the use of capacitive division provides a more effective means of reducing the switch voltage, since it requires a smaller trade-off with tuning range for a given switch voltage reduction. However, unlike the method of limiting $\theta_{\mathrm{C}}$, greater circuit area is required. Note that it is not necessary to impose the condition $\mathrm{C}_{1}=\mathrm{C}_{2}$; a different ratio and tuning range may be chosen as other requirements dictate.

Note that this analysis assumes that the switch is lossless, whereas a practical switch must have some DC resistance, resulting in a lower Q factor and therefore lower peak switch excursion voltage. Consequently, the achievable tuning range of a practical implementation may be slightly larger, however it is preferable to reduce such losses in order to maintain high $\mathrm{Q}$ and therefore larger magnetic field for power transfer.

\section{AdAPTIVE TUNING Using Zero-Voltage SWITChING OF FRACTIONAL CAPACITANCE}

Whilst existing methods of tuning LC circuits require amplitude measurement, the zero-voltage switching method inherently provides a method for determining if the circuit is ideally at resonance. In the foregoing discussions, it was assumed that the switch opening and closing instants were set to be symmetrical around the peak of the capacitor voltage when at resonance. This requires that the switch timing reference has an effective $\pi / 2$ phase relationship with the excitation. Therefore, the switch timing is defined under ideal conditions, otherwise if the voltage across the switch is not zero at the switching moment, the circuit is not at resonance.

If the modified resonant frequency is too low (figure 6a), the switch voltage excursion will not have returned to ground when the switch is closed. If the modified frequency is too high (figure $6 \mathrm{~b}$ ), the switch voltage drops below zero. ${ }^{\mathrm{TM}}$

The tuning error condition can be determined by comparing the sign of the switch voltage at its peak with the voltage sign just before disconnecting, to establish if there has been a reversal. Not only does this detection method indicate the resonant condition of the LC circuit, but a single observation of the switch voltage is enough to indicate the 
direction of the tuning error. The peak switch voltage sign is known directly from the control signals, hence a single sample and hold cycle is needed to sense the voltage just before closing the switch. Information about the resonant condition is available twice per cycle, thus it may be continuously regulated without disturbing normal system operation. Furthermore, a sign-based detection method eliminates dependence on the magnitude of the switch voltage, which will vary over the tuning range due to changes in $\mathrm{Q}$ factor with frequency.

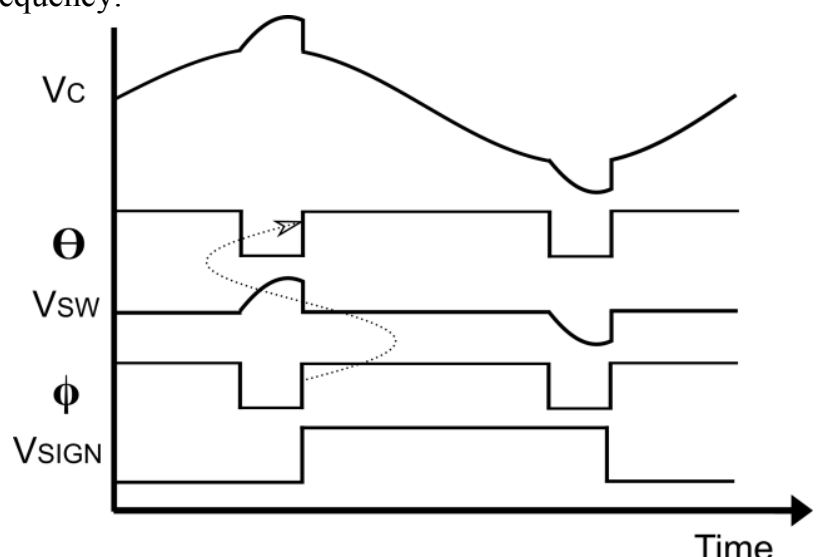

Fig.6a: Driven tank capacitor and switch voltages when the modified

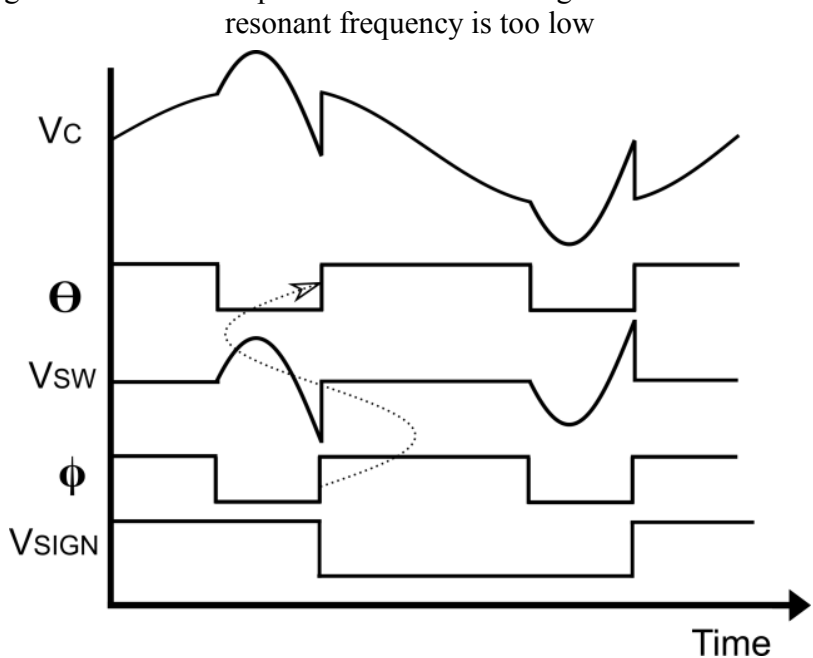

Fig.6b: Driven tank capacitor and switch voltages when the modified resonant frequency is too high

Other methods of detecting the error sign from the switch voltage are also possible. Figure 7 shows an example of a sample and hold system, based on tuning error detection. A unit value up/-down error signal is integrated to create the $\mathrm{V}_{\text {TUNE }}$ voltages used for timing generation.

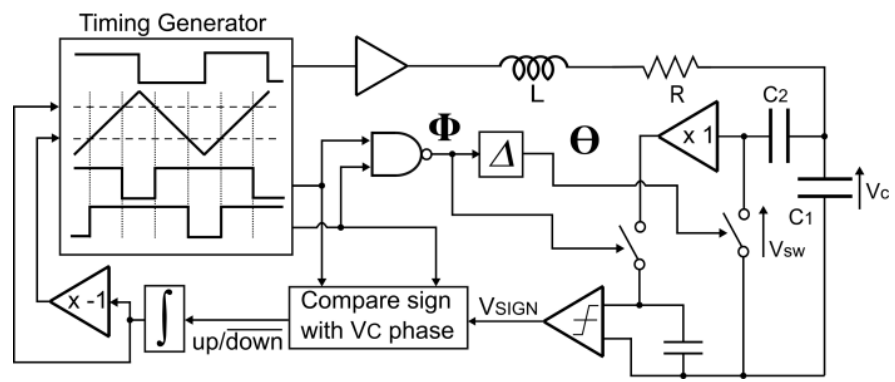

Fig. 7: Block diagram of self-tuning by sampling of vsw(t)
A convenient way of ensuring that the error sample is taken immediately prior to the switch closing is to insert a small delay between the control logic and the capacitor switch. Strictly speaking, this delay leads to a slight tuning error due to the asymmetry in the switch timing; however the effect is easily made negligible provided that this delay is much smaller than the period of oscillation.

\section{EXPERIMENTAL IMPLEMENTATION OF ADAPTIVE TUNING SYSTEM}

An experimental implementation of the adaptive tuning technique was constructed using standard discrete components for operation around $125 \mathrm{kHz}$, this range being commonly used in many wireless power systems. The tank circuit was realised with $\mathrm{L}=97.5 \mu \mathrm{H}$ (Q factor of 20), $\mathrm{C}_{1}=10 \mathrm{nF}$ and $\mathrm{C}_{2}=20 \mathrm{nF}$. An ADG452 CMOS transmission gate IC was used for the capacitor switch, with an excitation drive set to respect the breakdown limits.

The stored sign data were averaged and integrated using an analogue RC integrator, whose output voltage was used directly for the triangle comparator $\mathrm{V}_{\text {TUNE+ }}$ and $\mathrm{V}_{\text {TUNE- }}$ voltages, closing the adaptive self-tuning loop. Figure 8 shows stable operation at $108 \mathrm{kHz}$. The circuit is visibly at resonance, since the excursions of $\mathrm{vsw}_{\mathrm{sw}}(\mathrm{t})$ start and end at $0 \mathrm{~V}$, and there are no discontinuities in $\mathrm{v}_{\mathrm{C}}(\mathrm{t})$. In this example, the $\mathrm{RC}$ integrator time constant was set to $2.64 \mathrm{~ms}$, which was sufficiently large to preventdithering of the up/ᄀdown signal from causingsignificant ripple in the $\mathrm{V}_{\text {tune }}$ voltages, thus ensuring smooth tuning.

Figure 9 shows the response to abrupt changes in the drive frequency to illustrate the settling behaviour, stepping between $92 \mathrm{kHz}$ and $148 \mathrm{kHz}$. Note that the RC integrator here has a much shorter time constant $(264 \mu \mathrm{s})$ to make the system's tuning behaviour clearer in the figure, resulting in some dithering of the $\mathrm{V}_{\text {TUNE }}$ voltages during the settled states. Environmental detuning effects, such as the introduction of ferrous objects in close proximity to the inductor, will typically occur at a low frequency compared to the frequency of the tank, hence practical tuning systems may use a higher $\mathrm{RC}$ integrator time constant to ensure smoother operation whilst maintaining near-resonance of the tank at all times.

At the moment that the drive frequency changes, the amplitude of $\mathrm{v}_{\mathrm{c}}(\mathrm{t})$ drops, since the circuit is now being driven away from resonance, and its shape becomes discontinuous due to the incorrect $\mathrm{V}_{\mathrm{TUNE}}$ levels at this moment. The tuning loop however immediately begins to adapt, driving $\mathrm{V}_{\text {TUNE }}$ towards the new levels and shifting the effective resonant frequency. The amplitude of $\mathrm{v}_{\mathrm{c}}(\mathrm{t})$ gradually increases until it is once again continuous and the circuit stabilises in the new resonant condition. Only small residual variations in the $\mathrm{V}_{\mathrm{TUNE}}$ voltages remain, caused by the relatively short integration time constant used for this illustration.

Across the tuning range, an average $\mathrm{V}_{\text {DRIVE }}-\mathrm{V}_{\mathrm{C}}$ phase error of 0.62 degrees was observed. Table 1 compares the overall performance of the constructed circuit with a hypothetical binary-weighted capacitor system for both an equivalent tuning resolution and with tuning accuracy to $3 \mathrm{~dB}$ bandwidth. In both cases, fractional capacitance provides accurate tuning whilst significantly reducing the number of capacitor switches. 

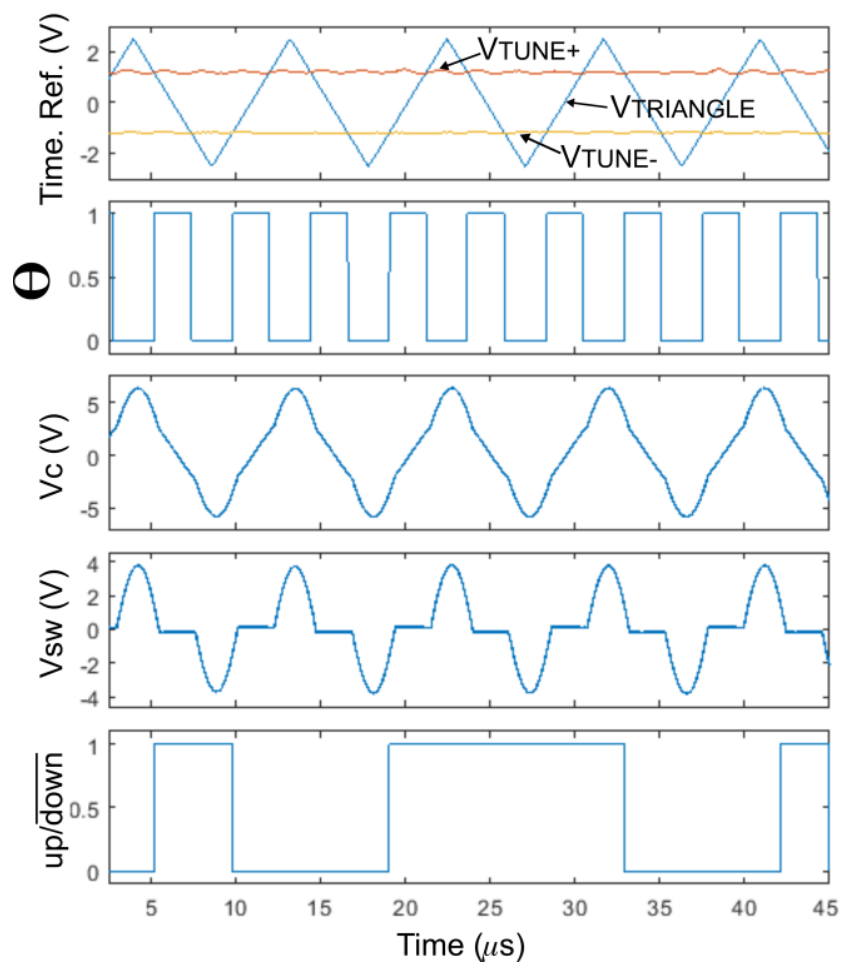

Fig. 8: Adaptive tuning: stable operation of experimental setup at $108 \mathrm{kHz}$ (tuning range $92 \mathrm{kHz}-148 \mathrm{kHz}$ )
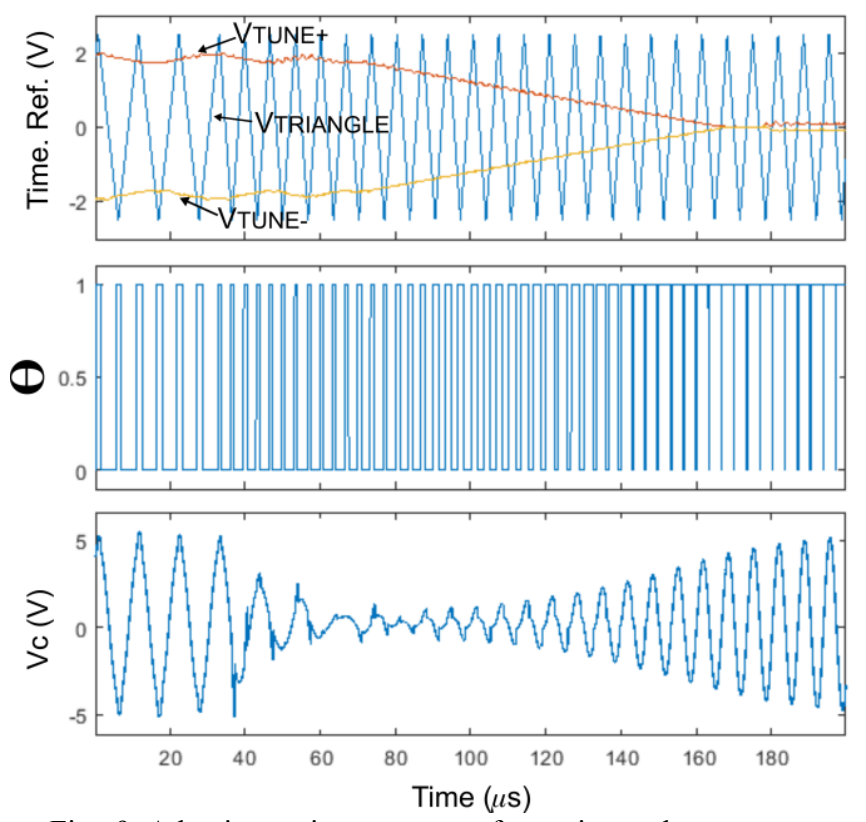

Fig. 9: Adaptive tuning response of experimental set-up to step change in excitation frequency $(92-148 \mathrm{kHz})$

\begin{tabular}{|c|c|c|c|}
\hline & \multirow{2}{*}{$\begin{array}{c}\text { Fractional } \\
\text { capacitance } \\
\text { measured results }\end{array}$} & \multicolumn{2}{|c|}{ Binary-weighted system } \\
\hline & & $\begin{array}{c}\text { Equivalent } \\
\text { performance }\end{array}$ & $\begin{array}{c}3 \mathrm{~dB} \\
\text { bandwidth }\end{array}$ \\
\hline $\begin{array}{l}\mathrm{V}_{\text {DRIVE }}-\mathrm{V}_{\mathrm{C}} \\
\text { phase error }\end{array}$ & $0.62^{\mathrm{O}}$ & $0.62^{\mathrm{O}}$ & $45^{\mathrm{O}}$ \\
\hline Switches & 1 & 11 & 5 \\
\hline
\end{tabular}

Table 1: Performance comparison between measured results and ideal binary-weighted capacitor tuning

\section{CONCLUSION}

An adaptive self-tuning technique for high-Q LC circuits in wireless power transfer systems and related applications has been presented. The method uses a zero-voltage switched fractional capacitor, providing a wide choice in tuning range for minimal additional cost, whilst maintaining the operational Q factor of the LC circuit. Methods for reducing the maximum switch operating voltage stress have been shown, by trading for tuning range, providing opportunities for further cost reduction. An architecture has been presented for adaptive tuning by observation of the switch voltage during the instant before it is closed. The adaptive tuning method is simple and economical to implement, and permits continuous tuning without interruption of the normal operation of the system, compensating for manufacturing and changing environmental effects that can affect tuning. Many variants of the technique are possible to suit different technologies and operating requirements. A discrete self-tuning $125 \mathrm{kHz}$ demonstration system has been constructed, showing a rapid tuning response to large instantaneous input frequency changes in a band commonly used by inductively coupled systems. Future research may include the development of an integrated circuit implementation, determining a suitable trade-off between available tuning range and peak switch voltages. More complex algorithms for adaptive tuning could be developed to reduce setting time whilst maintaining tuning stability.

\section{REFERENCES}

X. Zhu, S. K. Mukhopadhyay, and H. Kurata, “A review of RFID technology and its managerial applications in different industries," J. Eng. Technol. Manag., vol. 29, no. 1, pp. 152-167, 2012.

S. Li and C. C. Mi, "Wireless Power Transfer for Electric Vehicle Applications," IEEE J. Emerg. Sel. Top. Power Electron., vol. 3, no. 1, pp. 4-17, 2015.

R. R. Harrison, "Designing Efficient Inductive Power Links for Implantable Devices," IEEE Int. Symp. Circuits Syst., vol. 1, no. 2, pp. 2080-2083, 2007.

O. Jonah et al., "Optimal Design Parameters for Wireless Power Transfer by Resonance Magnetic," IEEE Antennas Propagat. Mag., vol. 11, pp. 13901393, 2012.

G. Covic et al., "Self tuning pick-ups for inductive power transfer," PESC Rec. - IEEE Annu. Power Electron. Spec. Conf., pp. 3489-3494, 2008.

6] M. Bruhnke et al., "Method for tuning an oscillating receiver circuit of a transponder built into a RFID system," U.S. Patent 6272321, 2001.

N. P. R. Hill, "Resonant Circuits," G.B. Patent GB2433381A, 2005.

J. James et al., "A Variable Inductor Based Tuning Method for ICPT Pickups," in Int. Power Eng. Conf., 2005, pp. 1142-1146.

H. H. Stover and G. L. Heiner, "System, circuit and method for tuning a resonant circuit," U.S. Patent 7190153, 2007. 